\title{
Strength and Conditioning Considerations for Elite Snowboard Half Pipe
}

\author{
Jonathon Turnbull ${ }^{*}, 1,2$, Justin W.L. Keogh ${ }^{2}$ and Andrew E. Kilding ${ }^{2}$ \\ ${ }^{I}$ New Zealand Academy of Sport - Winter Performance Programme, P.O. Box 395, Wanaka, New Zealand \\ ${ }^{2}$ Sports Performance Research Institute New Zealand, School of Sport and Recreation, AUT University, Private Bag \\ 92006, Auckland, 1142, New Zealand
}

\begin{abstract}
Snowboarding Halfpipe (HP) is a winter action sport which has progressed from being a recreational snow activity to a high performance snow sport such as traditional downhill or Nordic skiing. Like figure skating, gymnastics and diving, performance in the snowboard HP is subjectively assessed by a number of judges. The marking criteria focus on jump height (amplitude) and trick difficulty as the primary technical aspects. However, overall style and the appearance of effortless motion are also essential components of a well scored run. While HP performance is very technical in nature, considerable physical capabilities are required in order to maximize jump amplitude and remain injury free. This paper examines the scientific basis of the HP to highlight the role that sports scientists and strength and conditioners can play in this sport. Challenges that these practitioners may experience with these athletes are also discussed. Further research is required to characterize the physical capacities of elite HP snowboarders and how these compare to the stresses that training and competing may place on the human body. Such information may allow strength and conditioning coaches and sports scientists to develop more specific conditioning programs and to have a clearer understanding of the volume, intensity and mode of training athletes require and can tolerate in order to optimize their HP performance.
\end{abstract}

Keywords: Amplitude, eccentric, halfpipe, landing, snowboarding, takeoff.

\section{AN EMERGING PERFORMANCE SPORT}

Modern competitive snowboarding was born in the US in the 1960's. Since this time snowboarding as a culture and recreational pursuit has developed exponentially and is today one of the fastest growing sports internationally. Snowboarding's place as a performance sport was highlighted by its inclusion in the 1998 Nagano Winter Olympics. In relation to traditional winter sports of Alpine or Nordic skiing, the standard of performance in snowboarding may improve at a faster rate due to its infancy as an Olympic sport and the exposure it is getting as a competitive sport.

While this expected improvement will likely be multifactorial in nature, this paper postulates that strength and conditioning coaches and sports scientists can contribute substantially to this improvement.

Current practices of elite snowboarders appear varied in nature and there is often stark contrast between the practices of different teams. In saying this there appears to be a transitioning from very high volume but low specificity training (for example freeriding and skateboarding) to more purposeful strength and conditioning practices born in many instances initially out of ski racing programmes The purpose of this paper is to describe the biomechanical requirements of an elite snowboard Halfpipe (HP) rider that sports scientists and strength and conditioners may need to consider when working with these individuals.

*Address correspondence to this author at the New Zealand Academy of Sport - Winter Performance Programme, P.O. Box 395, Wanaka, New Zealand; Tel: +64 21 2227246; E-mail: Jon_T@xtra.co.nz

\section{WHAT IS SNOWBOARD HALFPIPE?}

Snowboard HP is one of two sub-disciplines of snowboarding (the other being Snowboarder-Cross) which have gained Winter Olympic status. A HP is a trough-type feature made either entirely of snow or with a base of shaped earth (Fig. 1). Competitive "runs" take around 20-30 seconds and involve 6-8 "hits" where tricks are performed. While a substantial anaerobic component likely contributes to each individual run, long repetitive days of hiking up the pipe in alpine environments and over long seasons also requires significant aerobic fitness [1, 2].

A typical HP run starts on the entry ramp several meters upslope of the pipe. The rider "drops in" from either side after leaving the entry ramp. The rider will drop into the eccentric transition (ET) just below the vertical (where the pipe wall is vertical), crossing the flat before entering the concentric transition (CT). The rider sets up for a trick as they enter the $\mathrm{CT}$, aiming to initiate the trick as close to the lip as possible. Once the rider is in the air, utilising the angular momentum gained by setting up in the CT, they will rotate through several axes about their centre of mass (CoM), depending on the trick. Tricks can be on or off axes (off axis tricks are termed 'corked tricks'), inverted, and on front side (toeside), or backside (heelside) during take-off or landing, depending on how the rider enters the pipe. Fig. (2) shows a typical takeoff, inversion, rotation and landing of a backcountry jump - similar to the positions of a halfpipe.

Snowboard HP contains areas of interest for many individuals in the sports science and medicine fraternities. Biomechanically, and from a motor learning perspective, HP 


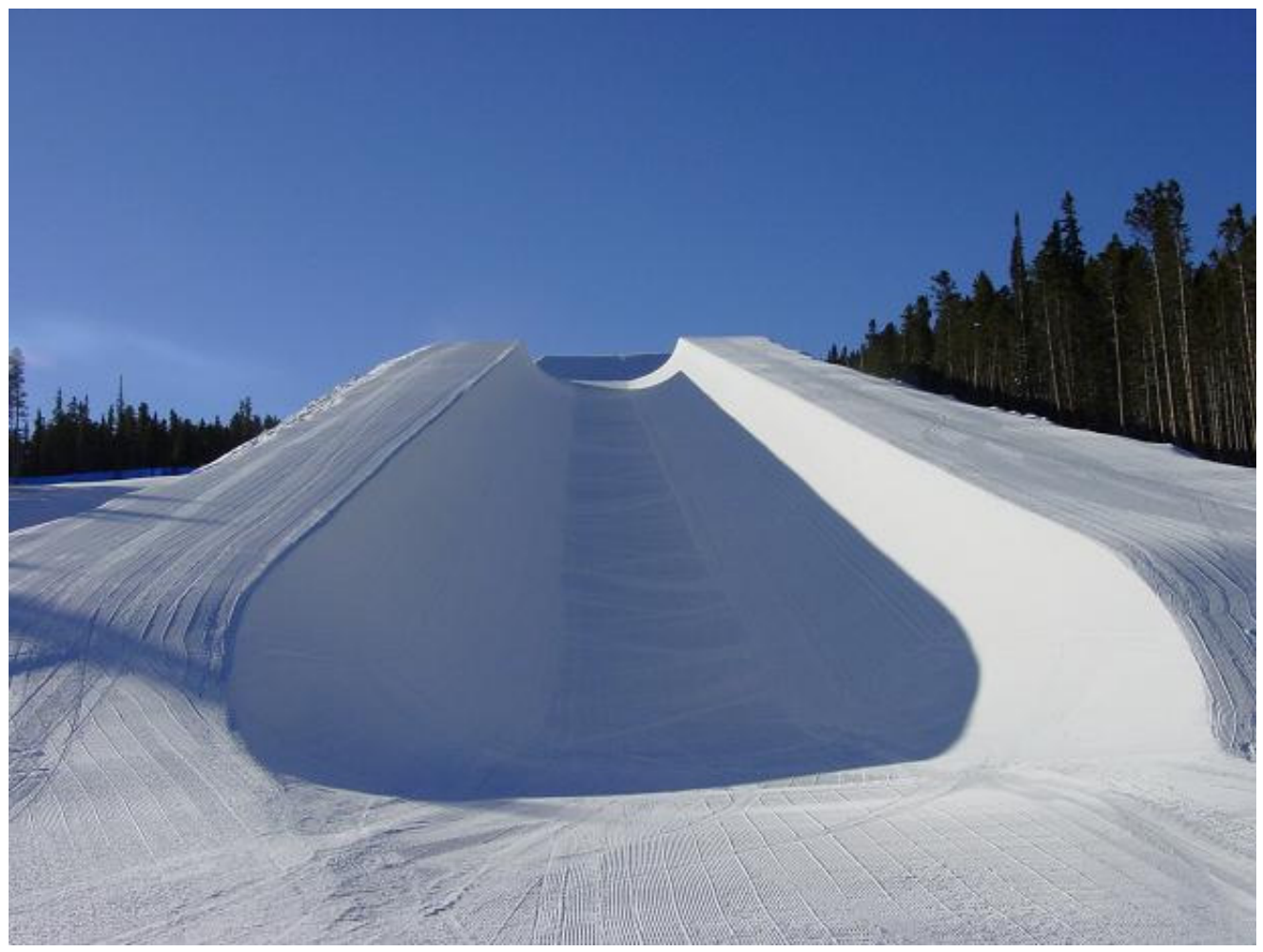

Fig. (1). Photographic and Diagrammatic Representation of a Snowboard Halfpipe. Halfpipe usually have a fall line gradient of 15-200. For the purposes of this review the Transition will be split in to the Eccentric Transition, where the rider drops into the pipe and sustains forces, and the Concentric Transition where the rider prepares to takeoff from the pipe sustaining isometric and/or concentric forces.

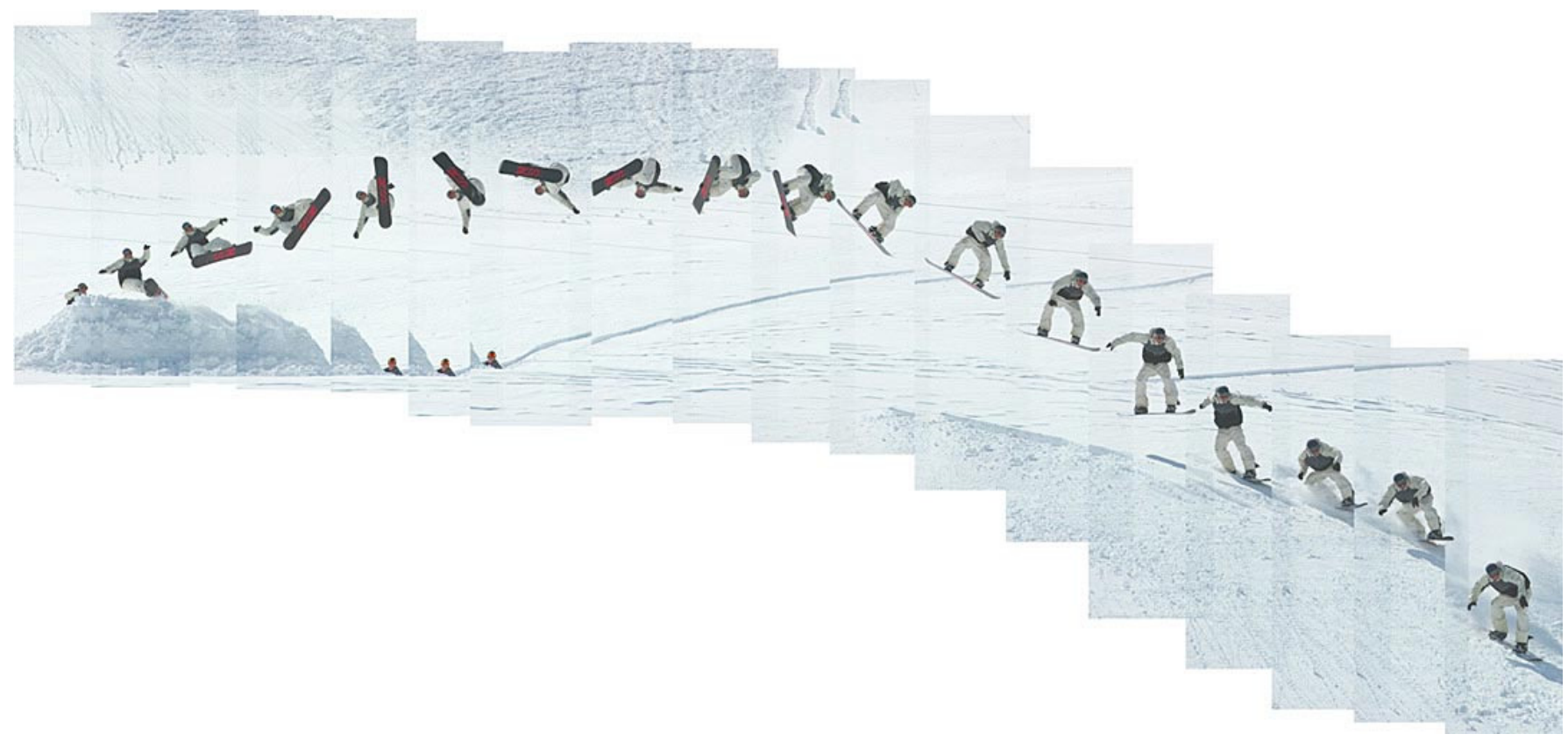

Fig. (2). Front 5' Melon trick off a backcountry jump. Melon Grab (front hand grabbing the heel-side edge behind the front foot and spinning 540deg counter clockwise.

is a skill-based event requiring significant kinaesthetic awareness and the absorportion and generation of force in a variety of "uncommon" anatomical positions. Riding positions incorporate significant knee internal rotation and adduction and ankle pronation. From an injury and conditioning perspective, landings occur from large heights and are often characterised by twisted and flexed spinal postures, factors that significantly increase spinal load [3]. The alpine conditions of HP and the length of the training day and season required to develop and refine technical skills would also make HP interesting to exercise physiologists. 


\section{Judging Criteria for Snowboard Halfpipe}

Similar to figure skating, diving and gymnastics, performance in snowboard HP lacks specific objective measurement. While each trick is judged based upon its place and difficulty in the run, tricks of significant amplitude, large rotation and fluid natural movement which are off the normal vertical axis (normal forces are those acting with gravity towards the horizontal), will generally be judged highly $[4,5]$. The judging criteria of the Federation International de' Ski (FIS) have also developed over the last 5 years to incorporate as much "rider expression" as possible. The most recent judging criteria is outlined in Fig. (3) [6].

\section{Direction and Quality of Literature: Previous Methods vs Current Trends}

Despite its rapid rise in recreational and competitive arenas, snowboarding HP has received very little attention in the scientific literature. Except for a very recent proliferation of injury reports, several computer game simulation discussions, and a small number of studies investigating HP physiology, little performance focussed research is evident $[2,3,7]$. It is possible that continued Winter Olympic participation will prompt further research into the performance aspect of the sport.

\section{KINEMATICS AND KINETICS OF THE HALFPIPE}

For the purposes of this paper, three key components of HP performance criteria are considered. Each component may determine a riders' scoring on the judging criteria outlined above:

1. Rider amplitude - based on how far above the lip the rider's CoM travels,

2. Trick difficulty - depending on the number of rotations and the addition of board grabs and other aspects which will assist "Overall Impression",
3. Successful landing - required for setting up subsequent tricks and preventing injury $[4,8]$.

Fig. (4) provides a simplified breakdown of the interaction of factors influencing trick success.

\section{Rider Amplitude - "Boosting and Maximising Air Time"}

Amplitude is important in itself as a judging criterion, but also for the ability to set up and execute tricks. Specifically, for a rider to have time to perform multiple rotations with control, their CoM must gain as much height above the lip as possible. Air time can also be gained by travelling horizontally down the pipe, however this sacrifices pipe space and may reduce the number of hits the rider can perform during a run. Several factors interact to assist and create amplitude.

\section{Board Control}

Efficient board control from the moment of landing determines the rider's ability to ride a line that optimises velocity across the pipe into the $\mathrm{CT}$ maximising takeoff velocity. Edge control and board trajectory are coordinated by unique movements outside the body's anatomical norm, including: forceful ankle pronation and large valgus knee angles for pressure and torsion on the board as well as significant lumbar and thoracic spine flexion and rotation in preparation for spins. The upper body provides gross movement for trajectory changes and trick initiation.

The magnitude of the various upper and lower body joint torques can differ considerably depending on where on the wall (i.e. low or high on the ET) the athlete lands and their position over the board on landing (i.e. toe side or heelside). These differences may be particularly large when comparing successfully completed tricks to ones that are missed, particularly if such a trick results in a crash.

\footnotetext{
Amplitude

Measures the height of the maneuvers. Amplitude is the distance measured from the lip of the pipe to the rider's center of mass. The amplitude score is derived from the sum of all hits, divided by the number of hits taken.

\section{Standard Airs (SA)}

These include all airs or tricks that are less than 360 degrees. The basic kinds of standard airs all grouped into one of the following: straight airs, air to fakie/fakie to forward, alley oop airs, straight switchstance airs, 180 handplants and liptricks less than 360 degrees.

\section{Rotations - Flat Spins (FS)}

These are all maneuvers that include a rotation of 360 degrees or more in a horizontal rotational plane (flat spins) including the rotations $(360,540,720,900,1080,1260$ and 1440$)$.

\section{Rotations - Inverts (IN)}

These are all maneuvers that include rotation of 360 degrees or more in a horizontal plane and over 180 degrees in the vertical plane in which the board breaks the vertical axis.

\section{Overall Impression}

The OI judges evaluate all phases of all the tricks. The judges will score the run by evaluating the run's overall precision, which includes the execution of the run, and the routine attempted no matter how the run is setup in its formation.
} 


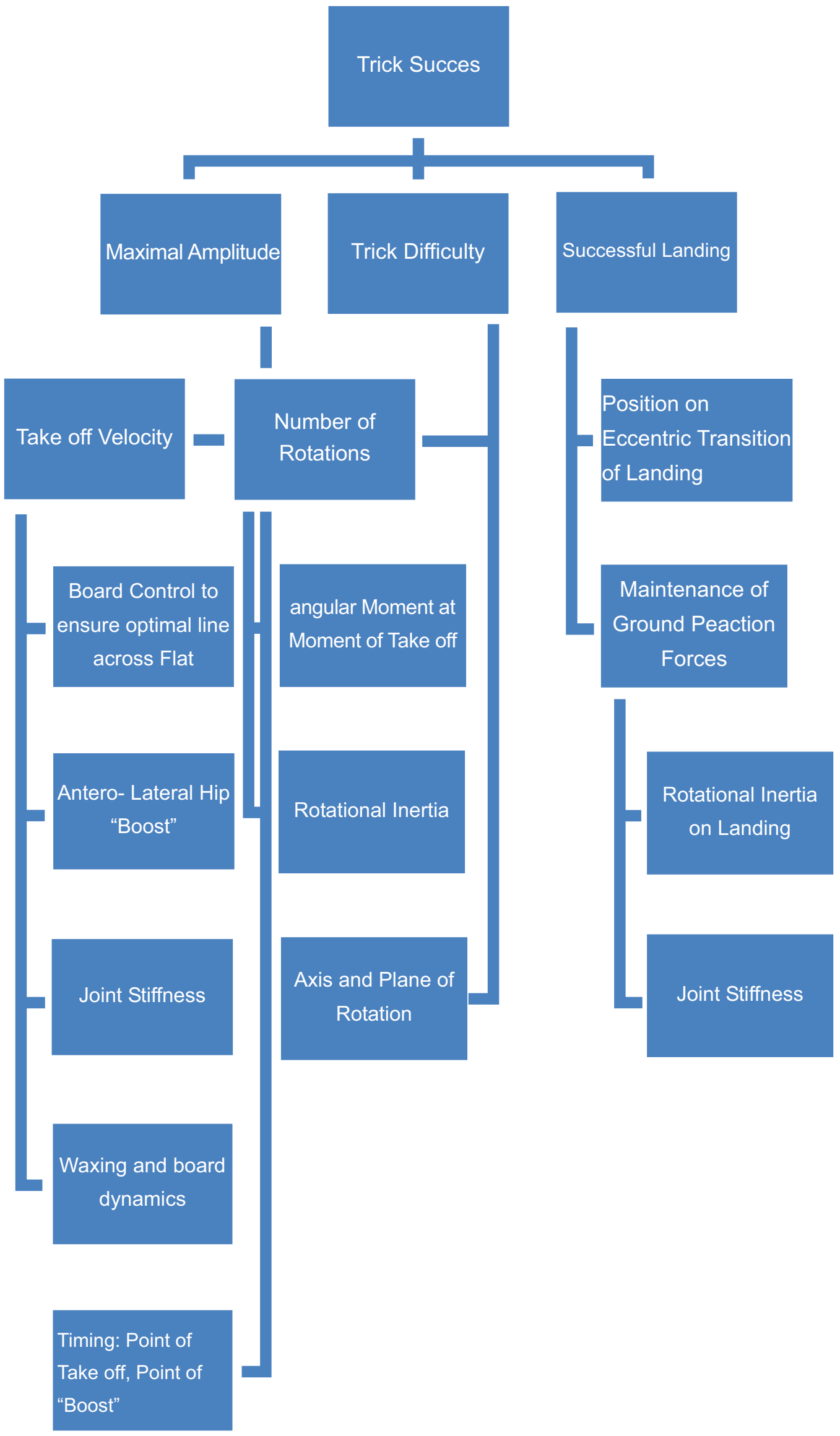

Fig. (4). Diagrammatic Representation of factors influencing performance in Snowboard Halfpipe. 


\section{Resisting Centripetal Force: Maintaining and Creating Horizontal Velocity}

Like other jumping sport, HP rider amplitude (i.e. jump height) is determined by the vertical velocity of the CoM at take off, which dependant on the horizontal in-run velocity, which in turn is affected by an efficient landing [8-11]. To maximize horizontal in-run velocity, riders aim to "stomp" their landings. "Stomping" entails minimising the absorption forces on landing by remaining stiff and immediately pushing down into the snow upon impact in order to increase the velocity on impact. To increase propulsive forces into and out of the ET the rider will "pump" the snow similar to a BMX rider [4, 12]. "Pumping" involves forceful lower limb extension when ground reaction forces (GRF) increase as the rider exits the ET onto the flat, thus applying pressure into the snow, allowing the force normal to the snow to cause more horizontal velocity.

\section{"Boost" and Take Off - Supplementing Horizontal Velocity}

To assist vertical velocity of the CoM at takeoff, rather than the vertical thrust of skaters, and other jumping sports such as basketball or volleyball, HP riders rotate their hips and knees forward towards the front of the board [4, 7]. From this position, anterior-lateral hip "boost" (a high impulse rear leg and hip extension toward the front of the board) and simultaneous lateral-vertical arm thrust (upwards and toward the front of the board) assist horizontal velocity (Note: horizontal velocity relates to velocity in the sagital or "cartwheel" anatomical plane) $[8,13]$. At the moment of takeoff, hip and arm movement is quickly ceased to combine the momentum of these segments with that of the rest of the rider and board [8]. This "boost" also allows the rider to align their CoM over the centre of the board which maximises the vertical component of their velocity, provides an axis to assist rotation and helps prepare the rider for landing [7]. If the rider extends vertically they risk propelling themselves too far into the pipe away from the ET, resulting in significantly greater GRF and increased injury risk. Impact force and landing position is discussed below.

\section{Trick Difficulty - Optimising Angular Momentum}

Successful high scoring tricks must be set up, executed and landed with control. Furthermore as part of the judging criteria relating to "Overall Impression", tricks must be original and seen to be clean and effortless without obvious precursory movement [6].

While overt biomechanical wind up and expression of effort is shunned by the culture and judging criteria, there needs to be some precursory movement to assist rotation [6]. Across the transitions and flat, the arms may be kept wide, increasing the moment of inertia to assist balance. To initiate spins, the rider will reduce their rotational inertia through a "wind up", as they enter the CT, forcefully rotating the arms from the wide position toward the CoM and the direction of desired rotation [8].

Once the athlete is in the air, their trajectory is predetermined as their angular momentum is conserved considering the only external forces on the system act at the $\mathrm{CoM}$, resulting in no external torques. The athlete therefore must alter the distribution of their body mass around the $\mathrm{CoM}$ to initiate specific rotational tricks. Rotational inertia may be reduced: by retracting arms toward the CoM for spinning (pirouette movement plane); by tucking the legs up, or crunching head and shoulders down for backward and forward flips (somersault movement plane), respectively. Combinations of spin and flip producing movements will produce high scoring off-axis tricks, but still involve the same biomechanical principles.

Approaching landing, the rider may increase their rotational inertia by "checking-out" or spreading the arms outward from the axis of rotation in order to maintain a linear path just as a gymnast does when dismounting [8]. As well as checking-out, upon landing a rider may increase the GRF and horizontal velocity by thrusting their arms downwards through the transition. This has a similar effect as pumping, or stomping in that it increases effective horizontal velocity.

\section{Successful Landing - Sticking it}

In HP, as with many gravity assisted sports, the significance of error and the risk of injury is most severe on landing [7]. Considering potential energy equals the product of mass, gravity and height, the greater the height of a previous trick the greater the PE which in turn will be converted to KE (mass $\mathrm{x}$ velocity ${ }^{2}$ ) on landing [14-16]. Maximising impact is not desirable for the rider per se, but is required to ensure maximal kinetic energy and resultant velocity when landing on sloped surfaces [15].

Considering the presence of the slope reduces the normal forces on landing, the impact the rider experiences is the force it takes to change the slope of their flight path to that of the HP ET wall and fall line $[11,15]$. These forces are dependent on: the amount of absorption in the legs on landing; how compact the snow is; angle of the ET wall and fall line (which is dependent on the position on landing); and horizontal velocity (relative to gravity and the fall line) of the rider prior to impact $[11,15,17]$.

The velocity vectors of landing on a sloped surface are outlined in Fig. (5). Considering the rider will generally be falling relatively vertically, the rider must land as high up on the ET, towards the vertical as possible with high joint stiffness. Reducing the stiffness of the landing [from high (vector 1) to low (vector 3)] will reduce the velocity at landing and hence increase the difference in Velocity vector $\mathrm{V}_{\mathrm{c}-\mathrm{b}}$. The smallest reduction in velocity (and consequently the least amount of impact felt by the rider) will occur where the rider's trajectory is as close as possible to that of the slope (as shown in vector 1, Fig. 5) when contact with the ET is made. In this situation the rider aims to "stomp" their landing increasing the impact velocity. Since the impact velocity trajectory and the slope angle are similar the stomp acts like a pump, increasing the rider's kinetic energy and hence speed into the next trick.

\section{Optimal Landing Positions - Maximal Kinetic Energy but Minimal Mechanical Stress}

Ideally, landing patterns for snowboard should closely resemble those observed during gymnastics "spiking" where the absorption of impact through large joint flexion must be constrained through well-timed joint stiffening to allow soft 


\section{(1)}

(2)

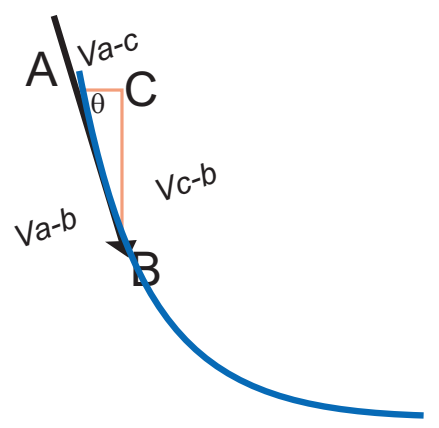

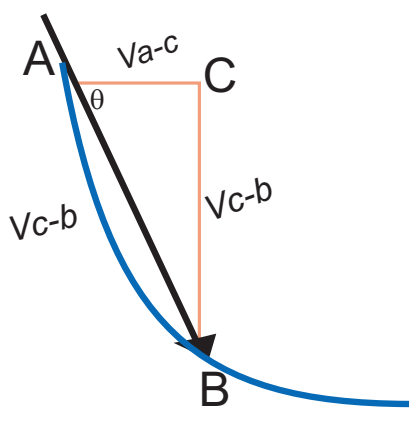

(3)

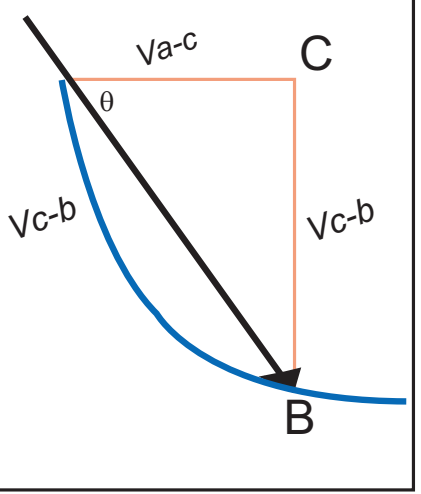

Fig. (5). Diagrammatical representation of Halfpipe landing. Point B is the riders' landing point while Line A-B is the flight path of the incoming rider, and Point $\mathrm{C}$ is the distance from the lip into the pipe. Angle $\theta$ is the angle of the rider's approach relative to the slope. The resultant reduction in velocity (identified by Line $\mathrm{V}_{\mathrm{c}-\mathrm{b}}$ ) is the difference between the rider's trajectory velocity and their velocity relative to the slope angle. Vector 1 represents an optimal high slope angle landing where impact forces will be minimal and may need to be assisted through "stomping" (forceful lower limb extension). Vector 2 and 3 represents progressively poor, flat landings and progressively near the end of the ET and the beginning of the HP floor.

tissue dissipation of forces [18]. An optimal landing will be high on the ET wall and involve high degrees of muscle stiffness to ensure maximum gravitational potential energy is converted to kinetic energy (velocity) of the rider.

Currently, there appears to be no peer-reviewed research which has reported the GRF inherent to landing from the HP. However, investigation into skateboard kinetics identifies a load of 4-5 times body weight (BW) from a skateboard Ollie height of less than $0.5 \mathrm{~m}$ [7]. Although the impact and absorption of snow and the angles and surface of the fall line and ET differ from the hard, flat concrete of skateboarding, HP riders often attain amplitudes of more than several metres above the lip, so that if landing some distance from the lip, they may fall a distance in excess of 3 metres. This may suggest that at least in landings that aren't high on the ET, HP riders may need the ability to tolerate very large GRF if they are to perform well and remain injury-free.

According to the impulse-momentum relationship, impulse needs to be created to change the momentum of the system. As the athlete has very high momentum on landing and has little time to dissipate GRFs, the athlete must produce high degrees of force very rapidly. The short contraction times required for a stiff landing will require a significant proportion of the rider's maximum voluntary contraction (MVC) force $[9,19,20]$. Further the stiffness of the system and the ability of the rider to tolerate the loading and landing positions are dependent on the skeletal posture and biomechanical alignment on landing. If the rider is aligned correctly over the board and hip, knee and ankle angles are at optimum force generating positions the rider will be able to withstand much greater forces and also produce much greater pump in order to gain speed through the ET.

Considering these factors, incorporating awareness of correct biomechanical position within specific strength training for HP athletes would appear essential to performance and injury prevention.

To assist in achieving substantial levels of muscle activity and isometric force which are required for gaining joint stiffness, HP riders, like skateboarders, should aim to pre-activate the lower limb musculature prior to ground contact [7]. Further increases in GRF can be developed by the athlete pumping their arm downwards and forward as they ride through the ET in order to transfer remote momentum from their limbs to that of their total body momentum and add to their horizontal linear velocity.

\section{Body Position, Joint Angles and Velocities and Muscle Activity}

Snowboarding and skiing are commonly mistakenly identified as "explosive" sports, which explains why coaches/trainers have put much time into training fast concentric movements in the past [21, 22]. However, in order to prescribe more specific training to these athletes, strength and conditioners need to have a good understanding of factors such as the body posture, joint angles and velocities, muscle activation patterns etc inherent to the HP. Berg et al. [23] and Berg and Eiken [24] have investigated how such characteristics may differ between the four ski disciplines. They found eccentric action was the prevalent muscle contractive force during ski racing [23, 24]. A number of these variables are summarized in Table $\mathbf{1}$.

Much of what is currently known regarding the biomechanics of skiing may be applied to snowboarding considering they are both gravity assisted and share similar snow surface and carving/turning mechanics. From initial observation, the joint angles and angular velocities associated with HP during the landing and trick initiation may be more in the range found for freestyle mogul skiing. However the duty cycle (time between turns/tricks) may be more in the range of Super Giant-Slalom skiing, considering the time to cross from ET to CT $[24,25]$. Thus, we have a 
Table 1. (L-R = Time Period from the Start of the Left Footed Turn (where the Left Ski is the Downhill Ski) to the Start of the Right Footed Turn. MVC = Maximum Voluntary Contraction, SL = Slalom, GS = Giant Slalom, SG = Super Giant Slalom, FM = Freestyle Mogul)

\begin{tabular}{|c|c|c|c|c|}
\hline & Outside Load Bearing Knee Angles $\left({ }^{\circ}\right)$ & Knee Angular Velocity $\left({ }^{*} \cdot \mathbf{s}^{-1}\right)$ & Movement (Duty) Cycle L-R (s) & Lower Body \%MVC \\
\hline \hline SL & $98-111$ & $69 \pm 11$ & $1.6 \pm 0.2$ & $74 \pm 33$ \\
\hline GS & $86-114$ & $34 \pm 2$ & $3.5 \pm 0.6$ & $73 \pm 21$ \\
\hline SG & $83-96$ & $\sim 17$ & $\sim 4.1$ & - \\
\hline FM & $62-133$ & $\sim 300$ & $\sim 0.8$ & - \\
\hline
\end{tabular}

scenario where HP riders experience greater maximal force and impulse on landing which are being held for a longer time, relative to competitive ski racing. Clearly, both of these factors need to be considered carefully when prescribing physical conditioning programmes.

Considering the magnitude of impact loads that are imposed on the HP rider (especially if the rider lands low on the HP wall and experiences a flat landing), the rider needs to distribute these forces across the musculoskeletal system if the injury risk to any particular anatomical structure is to be minimized [16]. Upon landing from a skateboard Ollie, electromyography studies reveal an oscillation in the activity of many muscles used to counteract vertical ground reaction forces, due to the rider adjusting their CoM vertically, sagitally, and laterally over the board. Similar oscillations are likely to occur in HP as the rider adjusts their CoM on landing in response to board position, fall line and snow surface features (such as holes, or bumps of hardened snow). Minimizing the range of oscillation however, will allow greater kinetic energy to be available to assist horizontal velocity, but will require specific sequencing and intensity of muscle activation in order to avoid significant shock loading $[14,26]$.

\section{CURRENT HALFPIPE TRAINING PRACTICES AND THE ROLE OF SPORTS SCIENCE AND STRENGTH AND CONDITIONING}

The following section of this paper focuses on the potential to improve performance and reduce injury risk in the HP, as well as some of the challenges that may arise when working with these athletes.

\section{Citius, Altius, Fortius - The Need for Sports Science}

Some coaches and many young riders are of the opinion that the only way of becoming a better snowboarder is to snowboard more [8]. While it may be argued that skill based competition is best trained by actually doing the activity, the need for "training to train" should be understood with respect to the speed of skill development. For example, while the high repetition work performed on snow may eventually develop sufficient strength and neural control required to successfully complete highly technical maneuvers (e.g. 10's and 12's and inverted maneuvers), spending the off-season building this strength through progressive resistance training, the ability to spin via trampolining and diving may allow more rapid developments from the time spent on the slopes. Furthermore ensuring a quality environment for learning must be taken into account. If sufficient fitness is lacking to remain mentally and neurally alert, decision making, reaction time and/or muscle activation patterns may be compromised resulting in poor adaptation and learning and potentially increased falls and injury risk.

Coaches and athletes must appreciate the physiological stresses that the riders are under during normal performance, and under injury precipitating mistakes [27, 28]. When they do, they may more easily accept the proven training methodologies of specificity, overload, and recovery. Using knowledge of the forces and energy systems characterizing $\mathrm{HP}$, the sport scientist or strength and conditioning coach will be better able to develop injury prevention strategies and, develop talent identification programs and sport specific fitness and technique assessments [27-31].

\section{The Sporting Context}

The technical nature of HP does mean that on-snow time is paramount to trick development [28]. Northern Hemisphere competition starts mid October, not ending until mid April the next year. The Southern Hemisphere training season may extend from June until October. Thus, limited time exists for dedicated 'off-snow' physical training for snowboarding. Consequently, training interventions must be short and intensive to fit between on-snow seasons/camps $[30,32]$. More importantly during the competitive season, training programs must be at a level that does not induce significant fatigue but still allows physiological development and does not impair the riders on-snow performance or increase their risk of injury, both of which will ultimately limit skill development and competitive performance [5].

\section{The Riders.... Athletes (?)}

As with any high level training method, a base level of conditioning is needed for injury prevention and in order for movement- and velocity-specific training to be effective [5, 19,33 . Snowboarding and more specifically HP is currently the realm of the young athlete, with many of the top riders in their teens or early 20's. As a result the athletes (and coaches) we are dealing with often have limited physical training history and training age. As a consequence of the sporting culture and self expression ethos of board sports, the athletes commonly have little inclination to do off-snow training, and even less understanding of the performance enhancing potential traditional training methodologies like strength and conditioning and sports science in general may have for their sport. Thus, an understanding and empathy of the culture of snowboarding and typical HP rider age is essential for sports scientists and conditioners who wish to work with these athletes. Without this trust and 
understanding, much time and resources may be wasted by both parties.

\section{Critical Considerations for the Strength and Conditioner in a skill Based sport}

\section{Performance Interventions}

Due to its importance in performance and injury prevention, the landing appears to be the component of snowboarding which strength and conditioners may most easily influence. However, to be of most use strength and conditioners must eventually consider the sport from start (drop-in) to finish (last trick). Interventions must direct training methods to facilitate optimal landings which, from a performance aspect, will allow the rider maximum chance of setting up their next trick, but also from an injury prevention perspective, will ensure the rider is robust enough to cope with the eccentric ground reaction forces and joint torques encountered during less than optimal landings [18].

\section{Maximising Amplitude}

On landing the rider aims to land on the uphill edge and sustain a position which will allow them to carve across and down the pipe to gain speed while maintaining balance. Strength and conditioning for creating joint stiffness and minimising braking forces and range of motion at the knee, hip and trunk in HP must also align with developing highintensity muscular endurance, balance and stability in low sustained and rotated postures. Berg and Eiken [24] found slalom skiers endured $75 \%$ MVC for duty cycle times of around 2 seconds during racing (Table 1). It is not unreasonable to suggest that HP riders would exhibit a greater range of maximum and minimum intensities over a longer duty cycle, given the GRF of the transitions, and the unloading which may occur through the Flat. Like figure skating and skateboarding, riders may ride, takeoff and/or land nose or tail first, and from either side (heelside or toeside). All four movement directions must therefore be considered by conditioners [4].

Using the impulse-momentum relationship, strength and conditioning for increasing horizontal velocity after landing should focus on the production of high levels of total body impulse (force multiplied by time). The overall impulse produced will reflect the angular and linear momentum of the trunk as well as the remote segmental momentum of the limbs, particularly the hip, knee and ankle joint moments that are produced in rotated and adducted femoral positions. Core strength is also needed to ensure that the transfer of remote momentum from the limbs is not dissipated through the trunk.

\section{Trick Difficulty - Spins, and Inverts}

Strength and conditioning for spins and inverts should focus on similar aspects used for arm drive to assist "boosting" from the pipe. As with arm movements, phasic core strength muscles must be adept at high impulse trunk rotation and extension to assist wind-up and connection on takeoff. Connection refers to when the torso muscles stiffen allowing the upper body and the lower body segments to combine their momentum. This allows a smooth coordinated body movement providing a clean form which can be easily seen and judged. These muscles must provide high impulse movements in all 3 planes of movement to account for natural, switch, front-side and backside spins.

Tonic paraspinal core muscles must be sufficiently strong to ensure the body segment positions required for minimising rotational inertia can be sustained, while also providing stability for board grabs which will gain higher scores for basic tricks. Tonic control of paraspinal muscles is also critical on landing where maximal leg stiffness must not be compromised by trunk collapse, and to ensure the forces induced by joint stiffness are distributed evenly throughout the active joints and not just the spine and pelvis.

\section{Sticking It - Landing Safely and Conserving Momentum}

Power and impulse are important variables in HP performance as most actions must be fast and require high forces over relatively short periods of time in order to maintain momentum [34]. Unique to snow-sports which are some of the few gravity assisted sports, is the dominance of high load eccentric power associated with landing [24].

From a conditioning perspective the landing can be likened to a stop jump or drop jump task; where horizontal and vertical motion is rapidly reduced in order to change the direction of movement [31]. Joint and ligament risk during these tasks increases if muscle activation strategies (timing, activation pattern and coordination of agonist, antagonist and synergist) are not optimal and optimal biomechanical alignment is not achieved [35]. This is especially relevant when one considers the valgus and internally rotated postures of the lower limb found in HP (as depicted in Fig. 6), and the potential for landing too low on the ET where the magnitude of the GRF will be much greater.

In order to create the degree of stiffness required in high force landing, the rider must recruit as many motor units as possible from as many muscle groups as possible at the correct time [36]. Maximal strength training provides a means of recruiting as many fibres simultaneously as possible, while eccentric power and drop jump training (outlined below) could play a significant role in training correct muscle activation timing and joint range patterns, although more research is needed on the specific angles and firing patterns found in HP riding.

\section{Body Position, Joint Angles and Velocities}

As discussed previously, in order to maximise GRF and GPE conversion to kinetic energy and effective velocity on landing, joint stiffness must be high and absorption through knee bend and hip-spine flexion limited if landing high on the ET. If a rider lands low however, they may need to absorb more force through a greater range of motion in order to reduce injury risk and regain balance. While the stance depth may not alter the GRF's during concentric or eccentric loading, the range and velocity of joint motion has a significant effect on the forces under which the knee is placed [31]. Joint stiffness incurs large angular velocity rates over small ranges of motion. Smaller knee angles (i.e. lower landing postures) do, however, relate to increased risk of anterior cruciate ligament (ACL) injury through reduced hamstring capacity, regardless of angular velocity. Thus while landing with too much knee extension may result in excessive compression forces, landing with too much knee 


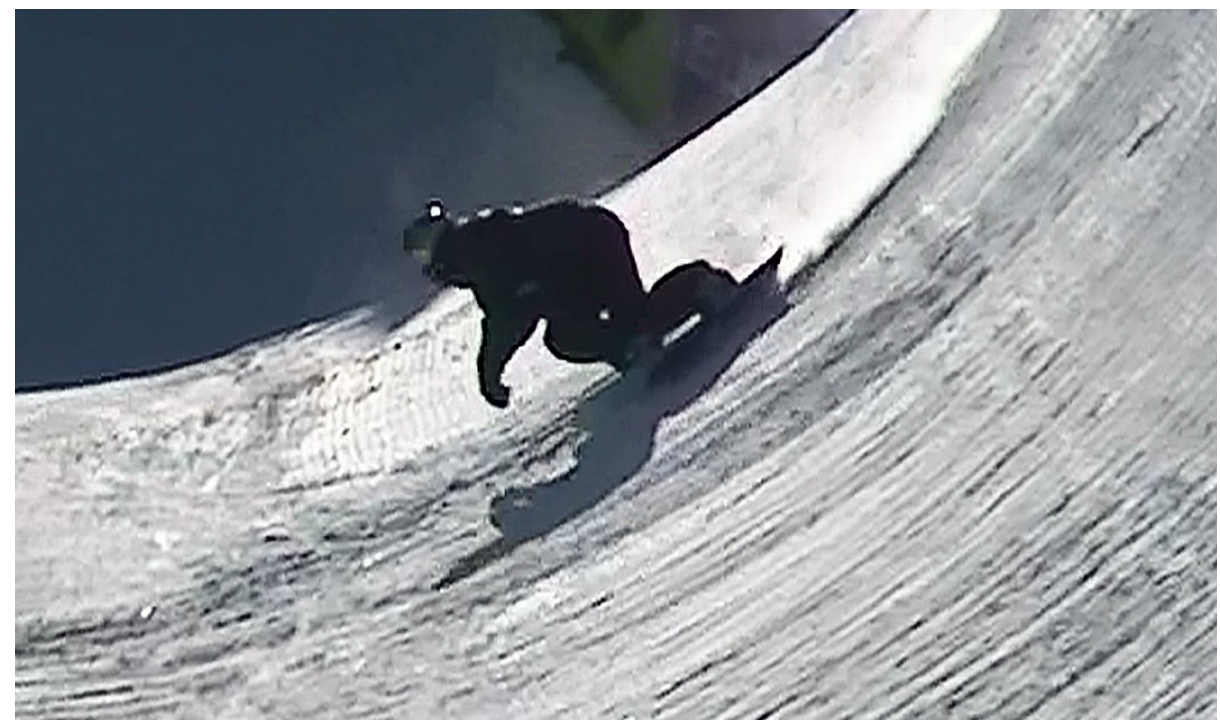

Fig. (6). Snowboard rider showing landing pattern typical of the ideal performance stance. Note the significant knee and ankle flexion and internally rotated back knee.

flexion may increase the risk of non-contact ACL injuries through ineffective hamstring contraction [31].

Conditioning exercises must therefore provide for a large repertoire of joint angles, loads and velocities to ensure riders are equipped to land safely in as many scenarios as possible. Identifying the range of joint angles, velocities, forces and torques involved in both poor and optimal landings is important in developing plyometric exercises which effectively simulate these landings.

\section{Injury Prevention Interventions}

\section{Crash Robustness}

Snowboarding has similar reported injury rates as skiing, with 4 injuries per 1000 rider days [37]. Most snowboarding injuries however, occur during jumping and landing impact, rather than when falling and experiencing hip and knee torsion during skiing $[3,22]$. Snowboarding exhibits significantly more ankle than knee injuries, specifically, anterior tibiofubular ligament strain and lateral talus (snowboarders ankle) fracture, compared to skiing or jumping sports such as volleyball or basketball [16, 37-39]. These ankle injury rates are greater in advanced relative to novice riders due to the fact that when more advanced riders fall, they typically do so from a greater amplitude. It has been proposed that one reason for such a predominance of ankle and impact injury in HP is due to the use of soft boots. Because these boots are built for a high degree of manoeuvrability in the HP, they provide limited support from impact and twisting [38]. The development of new boots that retain high levels of manoeuvrability but at the same time provide greater support appears important in this sport.

Shoulder dislocation and acromio-clavicular joint separation appears to occur across all levels of rider [37, 39], and are likely to be caused by blunt force trauma to this region resulting from a fall. Associated with these types of falls are wrist sprains and fractures, with novice riders tending to have a greater rate of wrist injuries than their more advanced peers.
It is the author's observation that snowboarders commonly exhibit high degrees of flexibility around the ankle joint in all planes of motion, with the ankle joint tending to be everted. Such characteristics may assist them in maintaining a feel for the snow and finding the internally rotated and adducted hip positions required of turning and take-off in the HP, however these postures are biomechanically inefficient for withstanding large forces. Strength and conditioning must focus on general robustness to endure crashes, with specific focus on shoulder and ankle joint strength. The rider must also retain the flexibility and feel of the ankle and hip joint to create the ankle and hip rotation required of fine board movements and trick initiation and landing.

Joint strength entails both good soft tissue support including muscle strength, ligament stability and muscle activation patterns [17]. Deep abdominal core strength and paraspinal muscle co-contraction is essential to tolerate the spinal loads inherent to landing and in transferring forces through the kinetic chain when preparing to perform a trick [40]. While retaining sport specific movements, to ensure biomechanical health the rider must be able to find and maintain neutral spinal positions when required.

\section{$\underline{\text { Seasonal Stamina }}$}

While a synopsis of the physiology of HP is beyond the scope of this paper, the physiological load and the fatigue developed through regular training through a season is a critical consideration for conditioners. For example an average training day may consist of 10-20 runs down and hikes up the pipe taking place over 2-4 hours. This level of riding may often be sustained for around 4-5 months during the Northern Hemisphere competitions period.

Further, although the physiological load of an individual HP run may not be great compared with other sports of similar duration (30-60 seconds), sports scientists must consider the temperature and altitude at which riders compete and live. Riders should be able to sustain numerous runs over several days, over months of competition, training 
and travel. All this takes its toll physiologically and psychologically, and as such must be considered in relation to aerobic development maintenance and recovery prescription to ensure that quality training occurs on snow.

\section{Specific Considerations of the Sporting Environment}

\section{Off-Snow Resistance Training}

With the exception of underweight athletes needing increased gravitational potential energy, resistance training protocols for HP riders must limit the hypertrophy response. This is due to the potential for increased body mass to reduce the vertical amplitude of the jumps and the ability to perform air-borne rotations due to an increase in rotational inertia. Furthermore, hypertrophy may speed the transition to slower myosin heavy chain isoforms, reducing the power potential of fast twitch fibres [10]. Increased body mass may also disrupt the riders kinaesthetic awareness while simultaneously placing greater stress on joints during landings [20, 29]. Instead, resistance training methodologies should focus more on neural adaptation such as motor unit recruitment, synchronization of agonist and synergist muscles, as well as eccentric modalities as discussed above $[19,41,42]$.

Skill aspects of riding, such as edge control, carving and speed awareness, and the "gymnastic" proprioception in the air requires many hours of practice to master. Trampoline, springboard diving and mini-trampoline to airbed/foam-pit are common training methods for trick development both inseason and during off-season dry land training. These methods are the closest riders can get to actual on-snow training and offer a safer environment for developing new and potentially dangerous tricks.

Combining sport specific movements with high load resistance training, may assist inter- and intra-muscular coordination. This has been shown to allow the smoothness and fluidity required of the skill activity to develop alongside strength and power qualities [41]. The optimal timing of these sport specific stimuli, post-activation potentiation or "tuning" activities is yet to be determined. However so long as loading (set/rep/rest) parameters excite but not excessively fatigue the neuromuscular system they should not interfere with the skill developing task [14]. These aspects should be considered when incorporating strength and trick development strategies such as trampoline and gymnastics training within the same training phase and/or training session.

When a given task is repeatedly performed, the central nervous system learns to create a pattern from the afferentefferent transformations The conditioner therefore needs to be mindful of negative transfer or the development of nonspecific and poor motor pattern in highly technical sports like the HP and regularly obtain feedback from the athletes and coaches to ensure that disruption of on-snow skills does not occur. In highly skill based sports such as the HP, it has been proposed that developing appropriate muscle activation patterns, timing, proprioception and balance may be more important than merely making the athlete stronger and more powerful $[12,19,34]$. As a consequence, whilst being sport specific is essential, athlete buy-in and understanding of the rationale behind prescription is important to make use of such specificity.

\section{FUTURE RESEARCH DIRECTION}

While there appears to be some literature on the HP relating to kinetics and kinematics in the sagittal plane, there is limited research into the frontal/lateral plane joint forces and muscle activation patterns, or with respect to intentional varus/valgus knee angles, and internal and external rotation of the hip and trunk, found in HP [18, 31]. However, there is even less data on the effectiveness or fatigue effects of common HP cross-training approaches such as trampoline, springboard diving, or mini-tramp training. Without specific and comprehensive data on HP performance and potential training strategies, developing and prescribing specific exercises for improving HP performance and reducing injury risk is severely limited.

The specific anthropometric, physiological, biomechanical and motor control profile of the 'ideal HP athlete' also requires quantitative investigation in order to assist conditioning practice, talent identification, appropriate fitness testing, and team selection criteria. As the sport progresses and more nations conduct testing and training interventions, more data will be available to create such performance profiles.

\section{SUMMARY AND CONCLUSIONS}

Snowboard HP is a rapidly developing performance sport for which very little scientific research has been performed to date. HP is judged through the quality of tricks performed during a run and the amplitude these tricks are performed at. Trick success depends largely on amplitude - the higher an athlete goes, the more time they will have for a trick to be executed. Amplitude is in turn dependant on the velocity of the in-run, which is effected by the efficiency of the landing of the previous trick.

Riders aim to minimize the potential for excessive impact forces by attempting to land high on the pipe wall, whilst ensuring significant joint stiffness to allow the potential energy of the high landing to be transferred as much as possible to horizontal velocity, rather than vertical impact force. If in the undesirable circumstance of a flat landing low in the transition, riders will need to use a large range of joint motion and a significant proportion of their MVC force to absorb the vertical ground reaction force which could lead to injury and or a fall.

Sports scientists and conditioners could play an important role in an injury prevention and performance enhancement capacity within this sport through more thorough investigation of kinetics and kinematics of HP riding. While the sport scientist and conditioner needs to understand the injury prevention and performance needs of snowboarding, they should also be aware of age and sport cultural issues regarding training motivation and history, and the physiological and time demands of on-snow training commitments.

\section{REFERENCES}

[1] Gabbett TJ, Domrow N. Relationships between training load, injury, and fitness in sub-elite collision sport athletes. J Sports Sci 2007; 21(13): 1507-19.

[2] Kipp RW. Physiological analysis and training for snowboard's halfpipe event. Strength Cond 1998; 20(4): 8-13. 
[3] Yamakawa H, Murase S, Sakai H, et al. Spinal injuries in snowboarders: risk of jumping as an integral part of snowboarding. J Trauma Inj Infect Crit Care 2001; 50: 1101-5.

[4] King DL. Performing triple and quadruple figure skating jumps: implications for training. Can J Appl Physiol 2005; 30(6): 743-53.

[5] Kovacs EJ, Bermingham TB, Forwell L, Litchfield RB. Effect of training on postural control in figure skaters: a randomised controlled trial of neuromuscular versus basic off-ice training programmes. Clin J Sport Med 2004; 14(4): 215-24.

[6] Federation Internationale de Ski F. FIS Snowboard Judges Manual 2006-07. www.fis-ski.com; 2006 [cited 2006 3/11/2006]; (http://www.fis-ski.com/data/document/fis-judgesbook-0607.pdf)].

[7] Frederick EC, Determan JJ, Whittlesey SN, Hamill J. Biomechanics of skateboarding: kinetics of the ollie. J Appl Biomech 2006; 22: 33-40.

[8] King MA, Yeadon MR. Maximizing somersault rotation in tumbling. J Biomech 2004;37: 471-7.

[9] Bobbert MF, Knoek van Soest AJ. Why do people jump the way they do? Sports Sci Rev 2001; 29(3): 95-102.

[10] Schmölzer B, Müller W. Individual flight styles in ski jumping: results obtained during olympic games competitions. Clin Biomech 2005; 38: 1055-65.

[11] Wylie DP. Who Hits Harder: The Nordic Skier or the Aerial Jumper? PUMAS Collection http:/pumas.jpl.nasa.gov 1999 [cited 2006 3/11/2006].

[12] Feltner ME, Bishop EJ, Perez CM. Segmental and kinetic contributions in vertical jump performed with and without an arm swing. Res Q Exerc Sport 2004; 75(3): 216-30.

[13] Virmavirta M, Kivekas J, Komi PV. Takeoff aerodynamics in ski jumping. J Biomech 2001; 34: 465-70.

[14] Bazzett-Jones DM. Effect of potentiation and stretching on maximal force, rate of force development, and range of motion. J Undergrad Res 2003; VI: 1-7.

[15] O'Shea MJ. Snowboard Jumping: newton's second law and the force on landing. Phys Educ 2004; 39(4): 335-41.

[16] Salci Y, Kentel BB, Heycan C, Akin S, Korkusuz F. Comparison of landing manoeuvres between male and female college volleyball players. Clin Biomech 2004; 19: 622-8.

[17] Strojnik V, Komi PV. Neuromuscular fatigue after maximal stretch-shortening cycle exercise. J Appl Physiol 1998; 81(1): 34450 .

[18] McNitt-Gray JL, Hester DME, Mathiyakom W, Munkasy BA. Mechanical demand and multijoint control during landing depend on orientation of the body segments relative to reaction force. $\mathrm{J}$ Biomech 2001; 34: 1471-82.

[19] Crewther B, Cronin J, Keogh J. Possible stimuli for strength \& power adaptation: acute mechanical responses. Sports Med 2005; 35(11): 967-89.

[20] Cronin JC, McNair PJ, Marshall RN. Power absorption and production during slow, large-amplitude stretch-shorten cycle motions. Eur J Appl Physiol 2002; 87: 59-65.

[21] Steadman RJ, Swanson KR, Atkins JW, Hangerman GR. Training for Alpine Skiing. Clin Orthop 1987; 216:34-8.

[22] Tarazi F, Dvorak FS, Wing PC. Spinal injuries in skiers and snowboarders. Am J Sport Med 1999; 27(2): 177-80.

[23] Berg HE, Eiken O, Tesch PA. Involvement of eccentric muscle actions in giant slalom racing. Med Sci Sports Exerc 1995; 27(12): 1666-70.
[24] Berg H, Eiken O. Muscle control in elite alpine skiing. Med Sci Sports Exerc 1999; 31(7): 1065-7.

[25] Petrofsky JSH. The interrelationship between blood pressure, intramuscular pressure, and isometric endurance in fast and slow twitch skeletal muscle in the cat. Eur J Appl Physiol 1984; 53: 10611.

[26] Haguenauer M, Legreneur P, Monteil KM. Influence of figure skating skates on vertical jumping performance. J Biomech 2006; 39: 699-707.

[27] Hartmann U, Mader A, Niessen M, Spitzenbfeil P, Lehnen J. Energy supply mechanisms in alpine ski racing - consequences for testing and training. In: Muller E, Bacharach D, Klika R, Lindinger S, Schwameder H, Eds. Science and Skiing II. Oxford: Meyer \& Meyer Sport (UK) Ltd; 2005. pp. 67-75.

[28] Neumayr G, Hoertnagl H, Pfister R, Koller A, Eibl G, Raas E. Physical and physiological factors associated with success in professional alpine skiing. Int J Sports Med 2003; 34: 571-5.

[29] Cronin JC, McNair PJ, Marshall RN. Velocity specificity, combination training and sport specific tasks. J Sci Med Sport 2001; 4(2): 168-78.

[30] Müller E, Benko U, Raschner C, Schwameder H. Specific fitness training and testing in competitive sports. Med Sci Sports Exerc 2000; 32(1): 216-20.

[31] Yu B, Lin C, Garret WE. Lower extremity biomechanics during the landing of a stop-jump task. Clin Biomech 2006; 21: 297-305.

[32] Bosco C, Cotelli F, Bonomi R, Mogononi P, Roi GS. Seasonal fluctuations of selected physiological characteristics of elite alpine skier. Eur J Appl Physiol 1994; 69: 71-4.

[33] McNitt-Gray JL, Requejo PS, Flashner H. Multi-joint control strategies transfer between tasks. Biol Cybern 2006; 94: 501-10.

[34] Pääsuke M, Ereline J, Gapeyave H. Knee Extension strength and vertical jumping performance in nordic combined athletes. J Sports Med Phys Fitness 2001; 42: 354-61.

[35] Beiser TF, Lloyd DG, Ackland TR, Cochrane JL. Anticipatory effects on knee joint loading during running and cutting manoeuvres. Med Sci Sports Exerc 2001; 33(7): 1176-81.

[36] Arampatzis A, Schade F, Walshg M, Bruggemann G. Influence of leg stiffness and its effect on myodynamic jumping performance. $\mathrm{J}$ Electromyogr Kinesiol 2001; 11: 355-64.

[37] Bindner SR, Greiger KM. The downside of snowboarding common injuries in novices and those seeking "hospital air". Postgrad Med 1999; 105(1): 83-8.

[38] Delorme S, Tavoulris S, Lamonatagne M. Kinematics of the ankle joint complex in snowboarding. J Appl Biomech 2005; 21: 394403.

[39] Zacharaopoulos AN, Tzanakakis N, Mitsou A. Injury types in skiing and snowboarding - the greek experience. In 15th ISSS Conference in Pontresina, Switzerland 27 April to 2 May 2003. Knee Surg Sports Traumatol Arthrosc 2004; 12: 169-77.

[40] Granata KP, Lee PE, Franklin TC. Co-contraction recruitment and spinal load during isometric trunk flexion and extension. Clin Biomech 2005; 20: 1029-37.

[41] Carroll TJ, Riek S, Carson RG. Neural adaptations to resistance training: implications for movement control. Sports Med 2001; 31(12): 829-40.

[42] Vikne H, Refsnes PE, Ekmark M, Medbo JI, Gundersen V, Gundersen K. Muscular performance after concentric and eccentric exercise in trained men. Med Sci Sports Exerc 2006; 38(10): 177081 . 